\title{
Wer sollte pendeln?
}

\section{Gerechtigkeitseinschätzungen von Mobilitätsarrangements in Partnerschaften von Doppelverdienern ${ }^{1}$}

\section{Von Thess Schönholzer}

Zusammenfassung: Doppelverdienerhaushalte befinden sich in einem potenziellen Mobilitätskonflikt, weil sie einen Wohnort und zwei Arbeitsorte koordinieren müssen. Dies wird besonders deutlich, wenn ein Partner seinen Arbeitsplatz an einen entfernten Ort verlegen möchte. In dieser Situation stellt sich die Frage, ob der gemeinsame Haushalt umziehen oder eine Person pendeln wird. In dem vorliegenden Beitrag wird diese Situation unter Rückgriff auf ein einfaches handlungstheoretisches Modell analysiert, das von zwei Partnern mit gegenläufigen Präferenzen und annähernd symmetrischer Verhandlungsmacht ausgeht. In diesem Rahmen kann das Mobilitätsdilemma nur gelöst werden, wenn eine Seite die eigenen Mobilitätspräferenzen jenen des Partners unterordnet. Kriterium für diese Entscheidung können soziale Normen z.B. über Geschlechterrollen in einer Partnerschaft bereitstellen. Hier werden zwei Typen betrachtet: Erstens generalisierte Geschlechterrollen, die auf der Idee des ,main breadwinners` beruhen, zweitens lebenslaufspezifische Vorstellungen, die die Existenz von Kindern berücksichtigen. Für die Frage, welche Normen wirksam sind, kommt eine Vignettenbefragung von Studierenden zum Einsatz. Die Probanden urteilen darüber wie gerecht sie Mobilitätsforderungen von betroffenen Partnern in bestimmten Situationen einschätzen. Wie sich zeigt, urteilen die befragten Männer und Frauen nicht grundsätzlich gemäß traditionellen Rollenbildern. Erst die Existenz von Kleinkindern bewirkt, dass die gesellschaftliche Norm ,primär sollen sich Frauen um die Kleinkindbetreuung kümmern', zum Tragen kommt. Diesen Frauen sollen nicht auch noch die Pendelkosten zugemutet werden.

\section{Problemstellung}

Der gesellschaftliche und wirtschaftliche Wandel der letzten Jahrzehnte hat in modernen Gesellschaften dazu geführt, dass mit raumplanerischen und verkehrstechnischen Massnahmen dem veränderten Mobilitätsverhalten seinen Bürgern Rechnung getragen wurde (vgl. Frick / Wüthrich / Zbinden / Keller 2004). Parallel lässt sich beobachten, dass immer mehr Frauen erwerbstätig werden (Bühler / Heye 2005). Zudem verfügen Frauen mittlerweile über vergleichbares Humankapital wie Männer und verfolgen ihre eigenen Karrieren. „Advanced societies all face a similar issue. The two-earner family has become the predominant form" (Gershuny / Bittman / Brice 2005: 656).

Für die Individualebene bedeutet diese Entwicklung, dass Paare auf die veränderten Mobilitätsanreize im Haushalt reagieren müssen. Tritt ein Partner eines Doppelverdienerhaushalts an einem entfernten Ort eine neue Stelle an, kann dies die gemeinsame Beziehung stark belasten. Soll der gemeinsame Haushalt umziehen oder wird der Stellenwechsler zum Fernpendler? Sofern die Partner auch in Zukunft zusammen wohnen wollen, stellt sie solch ein Mobilitätskonflikt vor eine schwierige Aushandlungssituation. Alleinstehende Personen, die an einem entfernten Ort ein neues Jobangebot annehmen, ziehen mit ihrem Haushalt meist in die Nähe des neuen Arbeitsortes (Ommeren / Rietveld / Nkjkamp 1997, 1998, 1999). In Paarhaushalten mit zwei erwerbstätigen Personen ist ein entsprechender Umzugswunsch für die stellenwechselnde Person aber nicht ohne weitere Absprache mit dem Partner möglich. Der Arbeitsweg der stellenwechselnden Person könnte sich durch einen Umzug zwar verringern,

1 Die Autorin bedankt sich bei Martin Abraham und Barbara Müller für die wertvollen Anregungen und das Korrekturlesen sowie den anonymen Gutachtern für die hilfreichen Hinweise. 
sein Partner hingegen müsste mit einem längeren Arbeitsweg rechnen oder über kurz oder lang ebenso eine Stelle in der Nähe des neuen Wohnortes suchen (Ommeren / Rietveld / Nkjkamp 1997; Clark 2003). Bei Doppelverdienerpaaren, in denen sich zudem zwei Personen mit vergleichbaren Arbeitsmarktressourcen gegenüberstehen, würde kaum ein Partner eine Mobilitätsentscheidung zu seinen Ungunsten hinnehmen. Ein Paarhaushalt mit zwei ebenbürtigen Partnern befindet sich von daher in einer Dilemmasituation, in der nicht klar ist, welcher der Partner im Mobilitätskonflikt nachgeben soll, damit sie als Paar eine zufriedenstellende Lösung finden.

In diesem Beitrag steht die Frage im Mittelpunkt, ob allgemeine Normen dazu beitragen, dieses Problem in Partnerschaften zu lösen. Zur theoretischen Analyse der Konfliktsituation wird ein allgemeines Modell aus der Spieltheorie - das sogenannte , battle of sexes ${ }^{6}$ - verwendet (vgl. Abraham / Schönholzer 2009). Zwei Partnern mit annähernd symmetrischer Verhandlungsmacht stehen zwei mögliche Lösungsstrategien zur Verfügung: Entweder wird gemeinsam an den neuen Ort umgezogen oder aber die stellenwechselnde Person pendelt. Aufgrund der gegenläufigen Präferenzen der Partner im Hinblick auf diese beiden Optionen sollte sich bei annähernd symmetrischer Verhandlungsmacht ein grundsätzlicher Konflikt ergeben, dessen Lösung ein Nachgeben einer Seite erfordert. Hier stellt sich die Frage, ob allgemeine gesellschaftliche Normen einen Lösungsmechanismus für diese Situation bereit stellen können. Die symmetrische Verhandlungsmacht könnte sich aufgrund von sozialen Normvorstellungen dahingehend verschieben, dass jener Partner nachgibt, der weniger zu verlieren hat, wenn er seiner gesellschaftlich zugedachten Rollen nachkommt.

Da vorerst nicht klar ist, woran derartige Normen geknüpft sein könnten, wird einerseits überprüft, ob auch für Paare mit annähernd symmetrischen Machtverhältnissen traditionelle Geschlechterkategorien eine Lösung bieten. Als sogenannte ,tied mover' oder ,stayer` wären es die Frauen, die ihre präferierten Mobilitätsarrangements nicht durchsetzen könnten, weil im traditionellen System des männlichen ,mainbread-winner" und weiblichen ,housekeeper' vor allem die Karriere der Männer gefördert wird (Mincer 1978; Bielby / Bielby 1992; Nivalainen 2004; Jürges 2005; Baldrige 2006).

Andererseits könnte aber auch vermutet werden, dass sich das Mobilitätsverhalten zweier ebenbürtiger Partner nicht per se mit einer traditionellen Geschlechternorm erklären lässt. Bei Partnern mit vergleichbarer Verhandlungsmacht könnten soziale Normen eher situationsabhängig zu einer Verschiebung der Verhandlungsmacht führen. Haben die Paare z.B. Kinder, könnte dies eine Einigung über Wohnstandort und Arbeitswege ermöglichen. Jene Person, die hauptsächlich für die Kinderbetreuung zuständig ist, sollte nicht auch noch mit zusätzlichen Pendelkosten belastet werden. Sind in einer Gesellschaft für die Aufgabe der Kinderbetreuung vor allem Frauen erwünscht, wären es die Väter, die ihre Mobilitätspräferenz nicht durchsetzen könnten und zum Ausgleich den größeren Anteil der Pendelkosten übernehmen würden.

Um die Auswirkung von gesellschaftlichen Normvorstellungen zu überprüfen, wird die in der Gerechtigkeitsforschung häufig angewandte Vignettenmethode verwendet (vgl. Beck / Opp 2001). Eine Zielsetzung der empirischen Gerechtigkeitsforschung ist es, festzustellen, inwieweit die in der Bevölkerung tatsächlich vorhandenen Gerechtigkeitsvorstellungen mit den theoretischen Konzepten in Einklang zu bringen sind und inwieweit sich hieraus Handlungen für das praktische Leben ableiten lassen (Liebig 2007). Als Ausgangslage für die Vignetten dieses Beitrags wurden jeweils zwei ebenbürtige Partner mit annähernd symmetrischer Verhandlungsmacht in einem gemeinsamen Haushalt skizziert. Durch Variationen in den Vignetten wurden verschiedene haus- und arbeitsmarktrelevante Situationen modelliert. Befragt wurden Studierende der Universität Bern. Die Probanden mussten die Situationen in den Vignetten dahingehend beurteilen, ob es mehr oder weniger gerecht ist, wenn in den vorgelegten Vignetten Männer oder Frauen die Pendelkosten zu tragen hatten. Mit dieser Erhebungsme- 
thode soll festgestellt werden, ob sich die Probanden beim Beurteilen der Mobilitätsdilemmata an gesellschaftlichen Normen orientieren. Wenn sich zeigt, wer in bestimmten Situationen pendeln sollte und wer nicht, wäre dies ein Hinweis auf soziale Normen in der Gesellschaft als Lösung des Mobilitätsdilemmas in Partnerschaften mit zwei erwerbstätigen Personen.

Der Beitrag ist folgendermaßen strukturiert: Zuerst wird auf die bestehende Literatur zu Mobilität in Paarhaushalten eingegangen. Insbesondere werden Theorie und Empirie zu Haushaltsumzügen, aber auch alternative Formen der regionalen Mobilität erörtert. Weiter sollen die bestehenden Erklärungsansätze mit der Modellierung aus der Spieltheorie ergänzt und mögliche Lösungsstrategien aus dem Entscheidungsdilemma vorgestellt werden. Anschließend wird das Untersuchungsdesign der Vignettenbefragung beschrieben. In der empirischen Analyse wird zuerst das Ausmaß des Mobilitätsdilemmas zwischen zwei Partnern mit symmetrischem Machtpotential dargestellt und in der Folge die formulierten Hypothesen als Lösungsmöglichkeiten analysiert und diskutiert.

\section{Stand der Forschung}

Wie sich zeigt, konzentriert sich die verfügbare Literatur zu Mobilitätsentscheidungen von Paaren fast ausschliesslich auf die Frage, ob ein Haushaltsumzug vorgenommen wird oder nicht. Bezüglich der innerhäuslichen Entscheidung, ob und gegebenenfalls welche Mobilitätsalternativen wie Fernpendeln, Zweitwohnsitze, oder Wochenendbeziehungen statt eines Umzuges in Frage kommen, ist hingegen nur wenig untersucht.

\subsection{Haushaltsumzüge von Paarhaushalten}

Am umfangreichsten stellt sich die Literaturlage zu der Frage dar, unter welchen Bedingungen Paare und Familien einen Haushaltsumzug durchführen. Eines der ersten theoretischen Modelle zu dieser Frage legte Mincer (1978) auf Basis einer haushaltsökonomischen Betrachtung vor, in der die Umzugsentscheidung von der Maximierung des gemeinsamen Haushaltsnutzens abhängt. Besteht die Möglichkeit, an einem entfernten Ort den Gesamtnutzen des Haushalts zu optimieren, kommt es zu einem Umzug, weil der gemeinsame Nettonutzen durch die Mobilität vergrößert werden kann. Haushalte werden gemäß Mincer dann umzugsmobil, wenn die Summe des individuellen Nutzens aller Haushaltsmitglieder die entsprechende Summe der Kosten durch die Mobilität übersteigt.

Dieses Modell, in dessen Rahmen die Partnerschaft als Entscheidungseinheit fungiert, wurde zunehmend mit dem Argument kritisiert, dass darin Interessenskonflikte zwischen den Haushaltsmitgliedern vernachlässigt werden. Stattdessen wurden Verhandlungs- und tauschtheoretische Modelle vorgeschlagen, welche die kollektive Mobilitätsentscheidung aus den individuellen Interessen der Haushaltsmitglieder zu rekonstruieren versuchen (vgl. Ott 1989, 1992, 1998). Hier antizipieren die Partner den drohenden Konflikt, den eine Umzugsentscheidung mit sich bringt. Wird der Konflikt im Sinne von drohenden Kosten als zu groß erachtet, wird ein Umzug vermieden (vgl. Kalter 1997, 1998; Auspurg / Abraham 2007). Die Beziehung zweier Partner wird dabei als Tauschverhältnis begriffen, dessen Tauschgewinne innerhalb der Partnerschaft verteilt werden müssen. Das Ergebnis dieser Verteilung steuert die relative Verhandlungsmacht der Tauschpartner, die durch externe Alternativen zur Partnerschaft beeinflusst wird. Je besser die möglichen Alternativen zu einer Tauschbeziehung für einen Partner, desto unabhängiger ist er von der Beziehung, und desto eher kann er oder sie mit der impliziten Drohung einer Beziehungsauflösung einen größeren Teil des Tauschgewinnes für sich beanspruchen. Diese Aushandlung setzt jedoch entscheidend voraus, dass die Partner (a) über 
Möglichkeiten für derartige Seitenzahlungen verfügen und (b) diese dann verbindlich erfolgen. Wie gerade die dynamische Verhandlungstheorie zeigt, ist letzteres ein zentrales Problem. ${ }^{2}$

Alternativ zu diesen eher ökonomischen Zugängen eines Umzugsentscheides wird zur Erklärung des Mobilitätsverhaltens auch die Rollentheorie verwendet (Bielby / Bielby 1992; Nivalainen 2004; Jürgens 1998, 2005; Baldridge / Eddleston / Veiga 2006). Während die oben erwähnten Ansätze Unterschiede zwischen den Partnern vor allem mit Effekten des Arbeitsmarktes erklären, verweist die Rollentheorie auf verinnerlichte Präferenzen, welche von Gesellschaftsmitgliedern mittels Sozialisation von einer Generation zur nächsten weitergereicht werden. Aus diesen normativen Vorstellungen resultieren Erwartungen an das Verhalten von Männern und Frauen, was in der Folge auch im Mobilitätsverhalten Geschlechterdifferenzen verursachen kann.

Im Folgenden sollen ausgewählte empirische Ergebnisse bezüglich der Umzugsentscheidungen in Paarhaushalten dargestellt werden. Ein erster, immer wieder bestätigter empirischer Befund ist, dass Doppelverdienerhaushalte generell weniger umzugsmobil sind als Haushalte mit nur einem Verdiener (Kalter 1998; Jürgens 1998, 2005). Im traditionellen System des männlichen ,mainbreadwinners' und weiblichen ,housekeepers' erfolgt z.B. ein Umzug meist dann, wenn dadurch die Männerkarriere gefördert werden kann (Bielby / Bielby 1992; Nivalainen 2004; Jürges, 2005; Baldridge / Eddleston / Veiga 2006). Dieses Ergebnis, dass Frauen tendenziell ,tied mover' sind, während Männer als ,mover' in ihre Karriere investieren können, bestätigt sich nicht in Partnerschaften mit Personen, welche über vergleichbare Arbeitsmarktressourcen verfügen. Auch in Beziehungen, in welchen die Partner gleichwertige Geschlechterrollen vertreten, unterscheidet sich das Umzugsverhalten von Frauen nicht von jenem ihrer Partner (Bielby / Bielby 1992; Jürgens 2005). Partnerschaften mit vergleichbaren haushaltsund arbeitsmarktrelevanten Kriterien finden sich zudem eher unter höher Gebildeten. Diese Gruppe zeigt zusätzlich weniger Umzugsmobilität im Vergleich mit weniger gut ausgebildeten Männern und Frauen (Jürgens 2005), wobei dieses Resultat nicht durchwegs bestätigt wird (vgl. Baldrige 2006). Neuste Studien zeigen, dass Frauen als Umzugsverliererinnen der Vergangenheit angehören und sie vermehrt als Gewinnerinnen aus einer Umzugsentscheidung hervorgehen (Nisic 2009). Dies wird mit der Angleichung des Bildungsniveaus von Frauen an dasjenige von Männern begründet (vgl. Jürgens 2005).

\subsection{Alternative Mobilitätsformen in Paarhaushalten}

Berufsmobilität ist heute nicht mehr gleichbedeutend mit Umzugsmobilität. Anstelle eines Umzugs gewinnen zum Beispiel Fernpendeln, Zweitwohnsitze oder Wochenendbeziehungen als alternative Mobilitätslösungen zunehmend an Bedeutung. Die von den statistischen Bundesämtern berichteten Zahlen über die abgenommene Anzahl der Haushaltsumzüge bestätigt diesen Trend (Carnazzi / Golay 2005; Statistisches Bundesamt 2002). Alternative Mobilitätsformen in Paarbeziehungen und Familienhaushalten sind aber weit weniger gut untersucht als Haushaltsumzüge. Schon hinsichtlich der Frage, in welchem Umfang derartige Mobilitätsformen auftreten, kann für den deutschsprachigen Raum praktisch nur auf eine einzige Studie zurückgegriffen werden. Die Ergebnisse von Schneider / Limmer / Ruckdeschel (2002) deuten im Allgemeinen darauf hin, dass verschiedene Alternativen von mobilen Lebensformen zu

2 In der klassischen Verhandlungstheorie könnten die Partner versuchen, sich gegenseitig Entschädigungen anzubieten. Dabei wird derjenige seine präferierte Mobilitätsform durchsetzen, der die höhere ,Zahlungsbereitschaft' aufweist. EGO kann ALTER z.B. eine höhere Beteiligung an der Haushaltsarbeit versprechen, dieses Versprechen kann ALTER jedoch kaum durch einen verbindlichen Vertrag absichern. Durch ,Seitenzahlungen' an den Partner dessen Zugeständnis zu erkaufen, ist demnach für rationale Akteure immer mit einem erheblichen Glaubwürdigkeitsproblem verbunden (Ott 1989, 1992, 1998). 
einem Umzug in Mehrpersonenhaushalten gelebt werden. Die Autoren gehen in ihrer Untersuchung für Deutschland davon aus, dass mindestens 16\% der Erwerbstätigen im Alter zwischen 20 und 59 Jahren in einer alternativen Mobilitätsform beruflich mobil sind. Die Mobilitätsform der Fernpendler wird dabei häufig gewählt, um eine Berufstätigkeit beider Partner zu ermöglichen (2002: 64),,wobei drei Viertel zu ihrem Einsatzort hin- die übrigen nach einem Umzug zum alten Arbeitsort zurückpendeln“" (2002: 101).

Dass gerade Fernpendeln als eine Alternative zum klassischen Umzug an den neuen Arbeitsort des Mannes gilt, zeigt auch die Langfristigkeit dieser Lösung. Kalter (1994) ${ }^{3}$ kommt in seiner Untersuchung zum Schluss, dass von den $2.5 \%$ Fernpendlern, die er als solche für Deutschland definiert, 64\% mindestens 5 Jahre, 46\% mindestens 10 Jahre und 29\% gar länger als 20 Jahre in dieser permanenten Mobilitätsform verharren. Als Grund, wieso Fernpendeln als Alternative zu einem Umzug gewählt wird, erwähnen sowohl Kalter (1994) als auch Schneider / Limmer / Ruckdeschel (2002) finanzielle Aspekte. Für Kalter ist Pendeln „ein ökonomischer Ersatz für Migrationen geworden“ (1994: 474) und für Schneider / Limmer / Ruckdeschel ist es „unter finanziellen Perspektiven die günstigste Mobilitätsform“(2002: 62). Berücksichtigt man hingegen die hohe psychische Belastung, welche mit Fernpendeln verbunden ist, wird keine andere Migrationsalternative als derart belastend empfunden wie das permanente Unterwegssein (Schneider / Limmer / Ruckdeschel 2002: 167).

Wie erwähnt, bezieht sich die verfügbare Literatur zu Mobilitätsentscheidungen von Paaren fast ausschliesslich auf die Frage, ob ein Haushaltsumzug vorgenommen wird oder nicht. Alternative Mobilitätsformen zu einem Umzug werden nur marginal diskutiert. Im vorliegenden Beitrag wird deshalb eine Verknüpfung beider Aspekte sowohl in theoretischer wie empirischer Hinsicht angestrebt.

\section{Ein einfaches Modell des Mobilitätsdilemmas in Partnerschaften}

Das zentrale Ziel des Beitrags ist es, zu untersuchen, ob im Hinblick auf den zurückgelegten Arbeitsweg allgemeine Normen existieren, die die Entscheidung von Paaren steuern können. Hierzu wird in einem ersten Schritt eine Situation modelliert, in der beide Partner die gleiche Ausgangssituation besitzen. Damit wird ausgeschlossen, dass aufgrund ungleicher Arbeitszeiten oder Einkommen die ökonomische Situation eine Lösung, diktiert‘. In einem zweiten Schritt wird diese Situation dann empirisch untersucht, indem von Probanden eingeschätzt wird, wie gerecht eine vorgegebene Entscheidung darüber ist, wer pendeln sollte. Dies erlaubt die Identifikation von allgemeinen Normen, indem der Einfluss von Faktoren wie z.B. Geschlecht des Stellenwechslers untersucht wird.

Das für den ersten Schritt verwendete abstrahierende Modell beschreibt Doppelverdienerhaushalte, in welchen zwei Partner je einen Arbeitsplatz und einen gemeinsamen Wohnort koordinieren müssen. Einer dieser Partner möchte an einem entfernten Ort eine neue Arbeitsstelle annehmen, da das Arbeitsangebot eine klare finanzielle Verbesserung gegenüber der aktuellen Stelle darstellt. Eine erste einschränkende Annahme ist, dass sich in der Ausgangssituation die Partner in möglichst wenigen Kriterien unterscheiden. Frauen weisen zunehmend ein vergleichbares Humankapital auf wie Männer. Zudem verfolgen Frauen vermehrt ihre eigene Karriere und stehen deshalb am Ende des Entscheidungsprozesses nicht mehr zwingend als Mobilitätsverliererinnen da (Bielby / Bielby 1992; Jürgens 2005). Das heißt, die Darstellung der Konfliktsituation bezieht sich auf zwei Partner mit gemeinsamem Wohnsitz und ver-

3 Die Untersuchung von Kalter (1994) benutzt Daten des SOEP, die in Deutschland jährlich über den Zeitraum von 1984 bis 1990 erhoben wurden. Es handelt sich somit nicht um eine Paarbefragung, sondern um die Einheit der Haushaltsgröße. Als Fernpendler definiert er Personen, die für einen Arbeitsweg mehr als $50 \mathrm{~km}$ zurücklegen. 
gleichbaren haushalts- und arbeitsmarktrelevanten Kriterien. Beide Partner sind zu gleichen Stellenprozenten angestellt und haben regelmässige Arbeitszeiten. Die Hausarbeit zwischen den Partnern ist paritätisch aufgeteilt. Als weitere Einschränkung gilt, dass Pendeln als einzige alternative Mobilitätsform zu einem Umzug angenommen wird. Da die Partner auch in Zukunft zusammen wohnen möchten, kommt weder ein Zweitwohnsitz noch eine Fernbeziehung in Frage. Zudem wird auf die Kompromissmöglichkeit verzichtet, dass ein Wohnsitz in der Mitte beider Arbeitsplätze gewählt wird. Die Pendelkosten werden also nicht aufgeteilt, sondern müssen von einem der beiden Partner hauptsächlich getragen werden.

Über die Präferenzstruktur der Partner wird folgendes angenommen. Beide Partner orientieren sich für ihre Mobilitätsentscheidung an den jeweils eigenen Mobilitätskosten respektive ihren eigenen Nutzen.

Dies sind zum Beispiel die Dauer des Arbeitsweges oder die alternativen Arbeitsmarktchancen zur momentanen Arbeitsstelle. ${ }^{4}$ Zudem wird angenommen, dass der Partner, der eine neue Stelle antreten möchte, im weiteren EGO genannt, in die Nähe seines neuen Arbeitsplatzes umziehen möchte. ALTER hingegen, der Partner von EGO, möchte lieber, dass EGO pendelt. Diese Grundhaltung der individuellen Nutzenorientierung wiederspiegelt die Dilemmasituation der entgegengesetzten Präferenzen der Partner, in welcher jeder versucht, möglichst nahe an seinem Arbeitsort zu wohnen. ${ }^{5}$

Durch die oben beschriebene Modellierung stehen sich zwei Partner mit vergleichbarer Verhandlungsmacht gegenüber. Als Entscheidungssituationen verbleiben den Partnern zwei Möglichkeiten: Entweder ein gemeinsamer Umzug an den neuen Arbeitsort von EGO (mit der Möglichkeit, dass ALTER an den Ausgangsort zurückpendelt oder sich in der Nähe des neuen Wohnorts eine neue Stelle sucht) oder aber die Beibehaltung des aktuellen Wohnorts, wobei EGO an seinen neuen Arbeitsplatz pendelt. Verhandelt wird in der hier vorgestellten Mobilitätsentscheidung darüber, welcher der Partner letztendlich nachgibt und bereit ist, das kleinere Stück des ,Kuchens` zu akzeptieren. Die Beschränkung auf diese Situation erfolgt bewusst, um die Existenz und Wirkungsmechanismen von (geschlechtsspezifischen) Normen in der Partnerschaft untersuchen zu können. Betont werden soll an dieser Stelle, dass reale Entscheidungssituationen meist deutlich komplexer, dadurch aber auch erheblich schwerer empirisch zu erfassen sind.

Die Struktur der dargestellten Situation kann durch ein aus der Spieltheorie bekanntes, einfaches Modell beschrieben werden. Im sogenannten ,battle of sexes ${ }^{6}$ stehen den Partnern jeweils zwei mögliche Handlungsoptionen zur Verfügung, die zu vier potenziellen Ergebnissen in der Partnerschaft führen. Einigen sich die beiden Partner nicht auf eine gemeinsame Handlungsoption, entsteht ein Konflikt, der die Beziehung belastet und zu einer Trennung führen könnte (vgl. Mincer 1978). In Abbildung 1 entspricht dieses Ergebnis den ,schlechtesten Alternativen ${ }^{6}$ aller möglichen Lösungen mit einer Auszahlung ${ }^{7}$ von jeweils Null für beide Akteure. Für beide ist es daher besser, wenn sie sich auf eine gemeinsame Handlungsoption ei-

4 Nebst dem zeitlichen Aspekt geht es hierbei auch um die sozialen Kosten/Nutzen eines Umzugs, wie z.B. den unmittelbaren Zugang zum Freundes- und Bekanntenkreis.

5 Die empirische Arbeitsmarktforschung verweist auf die Tatsache, dass kürzere Arbeitswege generell zu schlechteren Arbeitsmarktplatzierungen führen. Dieser Aussage wird hier nicht widersprochen. Auch wenn lange Arbeitswege ökonomisch einen Gewinn darstellen, versuchen Individuen die Pendelzeiten zu verkürzen, weil lange Arbeitswege eine zeitliche und psychische Belastung darstellen (Abraham / Hinz 2005; Schneider 2002).

6 Das Kooperationsspiel ,battle of sexes' wird in der Regel dazu verwendet, Konfliktsituationen zwischen zwei Spielern darzustellen (vgl. Schelling 1960; Luce / Raiffa 1967; Voss 2001; Rieck 2006).

7 Die Zahlen in der Tabelle geben lediglich die Rangordnung der Präferenzen für jeden Akteur an und sind darüber hinaus in ihrer Höhe beliebig, solange diese Rangfolge nicht verändert wird (Holler 2009). 
nigen können. Dies erfordert jedoch, dass einer der beiden ,nachgibt ${ }^{\star}$ und die Pendelkosten übernimmt. Dies bringt die asymmetrische Auszahlung in Abbildung 1 zum Ausdruck, in der der Pendelnde eine geringere Auszahlung erhält als der Partner. Den Partnern stehen damit zwei mögliche Lösungen zur Verfügung.

Abbildung 1: Mögliche Ergebnisse ,Umzug versus Pendeln“

ALTER

\begin{tabular}{|c|c|c|c|}
\hline & & Kein Umzug & $\begin{array}{c}\text { Umzug } \\
\text { (Option eigenes Pendeln) }\end{array}$ \\
\hline \multirow[b]{2}{*}{ EGO } & $\begin{array}{c}\text { Kein Umzug } \\
\text { (eigenes Pendeln) }\end{array}$ & $\begin{array}{c}1 / 2 \\
\text { Pendeln EGO }\end{array}$ & $\begin{array}{c}0 / 0 \\
\text { Schlechteste Alternative }\end{array}$ \\
\hline & Umzug & $\begin{array}{c}0 / 0 \\
\text { Schlechteste Alternative }\end{array}$ & $\begin{array}{c}2 / 1 \\
\text { Gemeinsamer Umzug } \\
\text { (Option pendeln ALTER) }\end{array}$ \\
\hline
\end{tabular}

Unter den gegebenen Annahmen ist das ,battle of sexes' nicht dazu geeignet, direkt eine Vorhersage über die Mobilitätsentscheidung in Doppelverdienerhaushalten zu treffen. Es kann lediglich voraussagen, dass zwei gleichwertige Lösungsmöglichkeiten existieren. Weder EGO noch ALTER verfügen über je größere Verhandlungsmacht als der jeweils andere Partner. Ceteris paribus hat im Modell kein Akteur Grund, nachzugeben und eine geringere Auszahlung zu akzeptieren. Beide streben danach, das große Stück des Kuchens für sich zu gewinnen. ${ }^{8}$ Damit landen sie aber in einer Konfliktsituation, die für eine Lösung eine Entscheidungsregel zur weiteren Aushandlung erfordert.

\section{Die ,normative' Lösung des Mobilitätsdilemmas}

In diesem Abschnitt wird ein Lösungsweg beschrieben, der das kollektive Entscheidungsproblem mittels sozialen Normen zu lösen versucht (vgl. Elster1989, Kap XII). Die Verhandlungsmacht der Partner orientiert sich dabei an Normvorstellungen darüber, wer durch Nachgeben mehr oder weniger zu verlieren hat. Die Partner finden dann eine gemeinsame Lösung, falls in bestimmten Situationen Normen existieren, welche rechtfertigen, wer in welcher Situation die Pendelkosten zu tragen hat. Im konkreten Fall einer Mobilitätsentscheidung würde dies heißen, dass es von den Akteuren als gerecht empfunden wird, wenn ein Partner die Pendelkosten dann trägt, wenn der jeweils andere seiner zugeschriebenen ,Rolle' nachkommt. Eine derartige Gerechtigkeitsvorstellung impliziert, dass die Verhandlungsmacht eines Akteurs von der Verteilung der potenziellen Kosten einer möglichen Lösung abhängt. Je höher diese Kosten bei einem Verzicht auf die präferierte Mobilitätsform sind, desto eher wird sich ceteris paribus der Akteur unter Berufung auf eine , gerechte' Lösung durchsetzen können. Die Differenz zwischen den Zuständen am Ziel- und Ausgangsort bzw. die Bedingungen der Mobilität werden so zum Indikator für die relative Verhandlungsmacht der Tauschpartner. Die Akteure bewerten verschiedene vor- und nachteilige Dimensionen der Situation für beide Seiten und versuchen, diese ,gerecht' in die Entscheidung einfließen zu lassen. Im Mittelpunkt der folgenden Überlegungen steht dabei die Annahme, dass diese relevanten Gerechtigkeitsvorstellungen zumindest partiell von gesellschaftlichen Normen beeinflusst sind. Dies könnte bedeuten, dass Vorstellungen darüber existieren, dass es nicht gerecht (oder anderweitig nicht

8 Um Missverständnisse zu vermeiden, soll hier nochmals betont werden, dass es sich um ein abstraktes Modell handelt, das der Komplexität realer Entscheidungen in Partnerschaften sicherlich nicht gerecht wird. In der Realität wird der Konflikt natürlich schon gelöst, weil die hier restringierten Möglichkeiten, wie zum Beispiel das Auflösen der Partnerschaft oder die Absage an das Jobangebot, in der Realität durchaus eine Option sein können. 
opportun) wäre, bestimmten Personen(gruppen) in spezifischen Situationen eine gewisse Mobilitätsform aufzuerlegen. Die anfänglich egalitäre Verhandlungsmacht der Partner wird dadurch asymmetrisch und das Nachgeben in der Entscheidungssituation für einen Partner zur Pflicht. Für eine konkrete Lösung des Mobilitätsdilemmas müssten sich in einer Gesellschaft allgemeine Vorstellungen darüber entwickelt haben, ob eher EGO oder ALTER hinsichtlich ihrer Mobilitätspräferenzen nachgeben sollte. Da vorerst nicht klar ist, woran derartige Normen geknüpft sind, werden in den nächsten Unterkapiteln zwei Möglichkeiten ${ }^{9}$ beschrieben, wie zwei annähernd ebenbürtige Partner mittels sozialen Normen das Mobilitätsproblem lösen können.

\subsection{Traditionelle Geschlechternorm}

Für den ersten hier präsentierten Anwendungsfall könnten sich solche Vorstellungen insbesondere am Geschlecht von EGO bzw. ALTER orientieren. ${ }^{10}$ Wird von Männern und Frauen ein gewisses Mobilitätsverhalten erwartet, kann dies in der Folge auch in der Mobilitätsentscheidung Geschlechtereffekte verursachen. Aus dem empirischen Forschungszweig über Arbeitswege ist bekannt, dass erwerbstätige Frauen grundsätzlich kürzere Wege zur Arbeit zurücklegen und weniger häufig lange Strecken pendeln als Männer (Johnston-Anumonwo 1992; Turner / Niemeier 1997; Clark / Huang / Withers 2003; Weinberger 2005). Dies kann als Hinweis darauf interpretiert werden, dass es geschlechtsspezifische Mobilitätsmuster gibt. Dahinter kann z.B. eine von der Gesellschaft akzeptierte Vorstellung stehen, dass Frauen eher durch Hausarbeit belastet sind, und es daher nicht gerecht bzw. nicht sinnvoll ist, ihnen auch noch längere Arbeitswege aufzubürden. In Partnerschaften mit klassischer Rollenteilung sind Frauen nebst ihrer (Teilzeit)-Erwerbstätigkeit hauptsächlich für den Haushalt zuständig. Männer hingegen gelten als ,main breadwinner', deren Erwerbstätigkeit ausschlaggebend für das Haushaltseinkommen ist. Bei einer Mobilitätsentscheidung kann sich jeweils der Mann durchsetzen, weil seine Karriere für den Haushalt wichtiger ist und deshalb mehr gefördert wird als jene der Frau (Bielby / Bielby 1992; Nivalainen 2004; Jürges 2005; Baldrige 2006). Die Kosten der Frau als ,tied mover' sind folglich geringer einzuschätzen als die zu erwartenden Pendelkosten für den Mann.

Vermögen traditionelle Rollenvorstellungen die Verhandlungsmacht von ebenbürtigen Partnern zu verschieben? In einer ersten Hypothese wird überprüft, ob auch Paare mit anfänglich vergleichbaren Verhandlungspositionen eine Mobilitätsentscheidung mittels einfacher Geschlechterzuordnung lösen. Ist EGO eine Frau, wird gemäß diesem theoretischen Argument der Mann seine Präferenz des momentanen Wohnstandortes durchsetzen und die Frau an ihren neuen Arbeitsort pendeln oder über kurz oder lang eine Stelle in der Nähe des neuen Wohnortes suchen. Ist EGO ein Mann, ist zu erwarten, dass er seinen Umzugswunsch durchsetzt, und die Frau an den Ausgangsort zurück pendelt oder sich in der Nähe des neuen Arbeitsortes vom Mann eine neue Stelle sucht. Es spielt also keine Rolle, ob die Frau die Rolle von EGO oder ALTER einnimmt. Männer werden als langfristige ,main breadwinner' nach dieser Regel von den zusätzlichen Pendelkosten entlastet. Damit würden sich im ,battle of sexes' immer die Männer durchsetzen. Die Annahme, dass auch annähernd ebenbürtige Partner bezüglich der Mobilitätsentscheidung von gesellschaftlichen Geschlechterrollenvorstellungen eines männlichen ,mainbreadwinners' und einer weiblichen ,housekeeperin “ beeinflusst sind, führt zu der ersten Hypothese:

9 Es sind durchaus verschiedene Möglichkeiten denkbar, wie das Mobilitätsdilemma durch die Partner gelöst werden kann. Als weitere Möglichkeit käme z.B. eine bestimmte Information über den Gegenspieler in Frage (vgl. Rieck 2006: 59).

10 Natürlich wären auch andere Zuordnungen möglich, für welche gesellschaftlich spezifische Normvorstellungen existieren. 
H1: Bei einem arbeitsmarktbedingten Mobilitätsanreiz eines Partners wird es generell als ungerecht empfunden, wenn der Mann die Pendelkosten tragen muss.

\subsection{Situationsabhängige Normen - Das Beispiel der Kinderbetreuung}

Allgemeine geschlechtsspezifische Normen können zwar theoretisch eine Lösung des Mobilitätsdilemmas darstellen, empirisch scheint jedoch zweifelhaft, ob vor allem in den jüngeren Generationen derartige allgemeine Wertvorstellungen zu finden sind (vgl. Abraham / Auspurg / Hinz 2009). Anders sieht dies aus, wenn Kinder in einer Partnerschaft vorhanden sind. Wie vielfache Befunde aus der Familiensoziologie zeigen, führt die Geburt des erstens Kindes zu einer erheblichen Umorganisation der partnerschaftlichen Aufgabenverteilung (vgl. Gornick / Meyers 2005; Peukert 2005). „Bei Doppelkarriere-Paaren folgt im Allgemeinen die Arbeitsteilung im Haushalt stärker dem Gleichheitsgrundsatz, aber es sind auch hier oft die Frauen, die die Vereinbarkeitsarbeit übernehmen“ (Burkart 2008: 199). Mit Vereinbarkeitsarbeit ist gemeint, dass Erwerbsarbeit mit Familienarbeit kombiniert wird. Frauen verfügen mittlerweile über eine vergleichbare Humankapitalausstattung wie ihre Partner. Wenn es jedoch um die Zuständigkeit für die Familienarbeit geht, scheint es eine gesellschaftliche Norm zu geben, die vorgibt, dass es vor allem Frauen sind, welche für die Kinderbetreuung zuständig sind.

Anders als bei traditionellen Normvorstellungen wird hier die Karriere der Frau aber nicht als geringer eingeschätzt als diejenige von Männern. Durch die Hauptverantwortung der Frauen für die Kinderbetreuung könnte es aber eher als ungerecht beurteilt werden, wenn Frauen auch noch die Mehrbelastung von langen Arbeitswegen zu tragen hätten. Dadurch verschiebt sich die Verhandlungsmacht im Mobilitätsdilemma. Die ungleiche Gewichtung, dass Frauen nebst der beruflichen Belastung auch noch hauptsächlich für die Kinder zuständig sind, bewirkt, dass Männer in dem Mobilitätskonflikt nachgeben. Diese Argumentation lässt sich auch spieltheoretisch abbilden. „In unserer Gesellschaft sind die Führungspositionen bevorzugt von Männern besetzt und der gesamte Umgang mit Kindern bevorzugt durch Frauen - ein Tatbestand, der ebenfalls als Ergebnis einer Koordinationssituation modelliert werden kann" (Rieck 2006: 67). Dabei spielt es keine Rolle, ob es sich bei den Frauen um EGO oder ALTER handelt. Ist die Frau in der Rolle von EGO, wird es eher als ungerecht beurteilt, dass die Frau die Pendelkosten trägt, weil sie nebst der Kinderbetreuung viel Zeit für Arbeitswege verlieren würde. Ist die Frau in der Rolle von ALTER, ist zu erwarten, dass es als gerecht eingeschätzt wird, wenn der Mann zu seiner neuen Arbeitsstelle pendelt. Auch hier spielt es also keine Rolle, ob die Frau EGO oder ALTER ist. Der Verzicht von Müttern auf ihre präferierte Mobilitätsform wäre im Vergleich mit demjenigen der Väter immer kostenintensiver. Kommen Frauen ihrer gesellschaftlich zugedachten Mutterrolle nach, werden unter Berufung auf eine ,gerechte ' Lösung die Pendelkosten immer den Vätern angelastet. Dies führt zu der zweiten Hypothese:

H2: Wenn in einer Partnerschaft Kinder vorhanden sind wird es bei einem arbeitsmarktbedingten Mobilitätsanreiz eines Partners als ungerecht empfunden, wenn die Frauen die Pendelkosten tragen müssen.

\section{Untersuchungsdesign}

Wie in der Einleitung erwähnt, wurde zur Überprüfung des Mobilitätsdilemmas eine Vignettenbefragung durchgeführt. Dieses Instrument, auch faktorieller Survey genannt, wird in der Gerechtigkeitsforschung immer wieder angewandt um soziale Normen zu analysieren (vgl. Beck / Opp 2001; Liebig / Mau 2002).

Die Erhebungsform einer Vignettenbefragung ist eine quasi experimentelle Messung und gilt als Alternative zu den klassischen Befragungsmethoden. Anstatt wie in eindimensionalen Befragungen nur ein Item abzufragen, werden in variierenden Vignetten komplexe Situationen 
mittels ,fiktiven' Darstellungen zur Beurteilung präsentiert. Die Probanden versetzen sich gedanklich in die Situationen und beurteilen die einzelnen Vignetten auf einer Antwortskala (für eine Beispielvignette siehe Abbildung 2). Es wird angenommen, dass das Antwortverhalten der Realität als alltagsnahe Rekonstruktion komplexer Entscheidungsprozesse sehr nahe kommt. Der Vorteil einer mehrfaktoriellen Vignettenbefragung liegt unter anderem darin, dass die Urteile nachträglich wieder in die einzelnen Dimensionen decodiert werden können. Auch wenn den Probanden selbst die Begründung für ihr Antwortverhalten nicht direkt zugänglich ist, können multivariate Methoden die komplexen Strukturen hinter den Urteilen wieder aufschlüsseln (Beck / Opp 2001; Auspurg / Hinz / Liebig / Sauer 2008).

Im vorliegenden Beitrag wird davon ausgegangen, dass in der Gesellschaft verankerte Normen bezüglich des Mobilitätsverhaltens auf die Mobilitätsentscheidung von Paaren in Doppelverdienerhaushalten einen entscheidenden Einfluss ausüben. Mit der Vignettenmethode werden diesbezügliche Entscheidungssituationen mittels Gerechtigkeitsvorstellungen der Probanden erhoben. Die Urteile, die von den Probanden über die Situationen in den Vignetten abgegeben werden, spiegeln dabei die gesellschaftliche Norm wider. Die Gerechtigkeitsvorstellung der Probanden äußert sich somit in ihrem Urteilsverhalten darüber, ob es mehr oder weniger ungerecht ist, wenn in den Vignetten Männer oder Frauen die Pendelkosten tragen.

Für das konkrete Vignettendesign dieses Beitrags wurde das im Theorieteil beschriebene Mobilitätsdilemma von Paaren in Doppelverdienerhaushalten modelliert. Die Ausgangssituation wurde bewusst so gewählt, dass die strukturellen Bedingungen für beide Partner möglichst ähnlich sind. ${ }^{11}$ Die verwendeten Dimensionen sind in Tabelle 1 dargestellt (vgl. Abraham / Schönholzer 2009). Zwei Variablen betreffen EGO, sein Geschlecht und seinen Arbeitsweg. Zwei Variablen beschreiben die Arbeitsmarktposition von ALTER am neuen Arbeitsort von EGO, darüber hinaus werden als gemeinsame Variable von EGO und ALTER die Anzahl der Kinder im Haushalt variiert. Die Merkmalsausprägungen sind auf maximal drei Werte beschränkt. So hat zum Beispiel die Dimension ,Pendelzeit‘ für einen einfachen Arbeitsweg die Werte $3 / 4$ Stunde, $1 \frac{1}{2}$ Stunden oder 3 Stunden (in der Beispielvignette von Abbildung 2 sind die variierten Werte in den Vignetten jeweils fett abgebildet). Multipliziert man alle möglichen Merkmalsausprägungen aller berücksichtigten Dimensionen, erhält man die maximale Anzahl möglicher Vignetten - das so genannte Vignettenuniversum. Dieses Vignettenuniversum umfasst im vorliegenden Design total 162 mögliche Kombinationen unterschiedlicher Vignetten. Aus dieser Gesamtzahl wurden 80 Vignetten gezogen ${ }^{12}$ und auf 10 verschiedene Decks aufgeteilt. Die Decks entsprechen den Fragebögen, welche den Probanden vorgelegt wurden.

11 Die Einleitung der schriftlichen Befragung lautete: „Zwei Partner wohnen zusammen in einem Haushalt, wobei die Hausarbeit zwischen ihnen zu gleichen Teilen aufgeteilt ist. Die Partner sind beide $100 \%$ erwerbstätig aber nicht selbständig. Einer der Partner möchte in Kürze eine neue Stelle antreten. Diese Stelle am neuen Ort beinhaltet 50\% mehr Lohn als bisher und bietet langfristig gesehen einige Aufstiegschancen. Der Arbeitsweg kann sowohl mit öffentlichen Verkehrsmitteln als auch mit dem Auto zurückgelegt werden. Infrastruktur, Kinderbetreuungs- und Freizeitmöglichkeiten sowie Wohnund Lebenshaltungskosten sind am neuen Ort mit denen am jetzigen vergleichbar. Der Stundenumfang der neuen Stelle entspricht der momentanen Erwerbstätigkeit. Eine Zweitwohnung am neuen Ort kommt aus finanziellen Gründen nicht in Frage".

12 Damit eine optimale Vermischung aller möglichen Merkmale gegeben ist, wurde diese Auswahl mittels fraktionellem Design gezogen (Dülmer 2007). Besten Dank an Katrin Auspurg von der Universität Konstanz für die Erstellung der fraktionierten Decks. 
Tabelle 1: Vignettendimensionen und Ausprägungen

\begin{tabular}{|l|l|c|c|c|c|}
\hline & \multicolumn{2}{|l|}{ Ausprägungen } \\
\hline & Dimensionen für EGO & $\mathbf{1}$ & $\mathbf{2}$ & $\mathbf{3}$ & Total \\
\hline 1 & Geschlecht & Frau & Mann & & $\mathbf{2}$ \\
\hline 2 & Pendelzeit einfacher Arbeitsweg & $3 / 4$ Std. & $1 \frac{1 / 2 \text { Std. }}{3 \text { Std. }}$ & $\mathbf{3}$ \\
\hline & Dimensionen für ALTER & & & & \\
\hline 3 & Chance auf Stelle & gering & Mittelmässig & gut & $\mathbf{3}$ \\
\hline 4 & Verdienstmöglichkeiten & niedriger & Vergleichbar & höher & $\mathbf{3}$ \\
\hline & Dimension als Paar & & & & \\
\hline 5 & Kinder im Haushalt & Nein & Ja, 3-jährig & Ja, 8-jährig & $\mathbf{3}$ \\
\hline & Vignettenuniversum & & & & $\mathbf{1 6 2}$ \\
\hline
\end{tabular}

Im Herbst 2008 wurden an der Universität Bern 356 Bachelor Studierende der Wirtschaftsund Sozialwissenschaftlichen Fakultät befragt. Wie die Literatur zeigt, leben vor allem höher gebildete Personen in egalitären Partnerschaften (Jürgens 1998, 2005). Die Auswahl der Kohorte von Studierenden scheint daher geeignet, weil viele selbst in einer Partnerschaft leben und sich darüber im Klaren sind, dass sie diese Situation aufgrund ihres nahen Arbeitsmarkteintritts in naher Zukunft selbst treffen kann. Jedem Befragten wurde ein zufälliges Deck mit 8 Vignetten vorgelegt. Die Aufgabe der Befragten war es, „Sich - soweit es geht - in die fiktiven Situationen hineinzuversetzen“, um dann aus Ihrer Sicht ein Gerechtigkeitsurteil abzugeben, welches Geschlecht in den Vignetten jeweils die Pendelkosten tragen soll. Die Probanden mussten sich also in die jeweiligen, fiktiven' Paare der Vignetten hineinversetzen und beurteilen, wie ungerecht eine Erwartung von ALTER an EGO ist, dass dieser pendeln soll. Das Geschlecht von ALTER und EGO in den Vignetten wurde dabei zufällig variiert. Dass sich die Studierenden gut in die beschriebenen Situationen hineinversetzen konnten, zeigt sich mitunter auch in der niedrigen Verweigerungsquote bei der Beantwortung der Vignetten. Nur maximal 9 Probanden beantworteten nicht alle 8 Vignetten. Weiter enthielt der Fragebogen einen zweiten Teil mit 6 demografischen Fragen zur Person der Befragten.

Abbildung 2: Beispielvignette und abhängige Variable

Variation 1 - Der Mann möchte die neue Stelle annehmen...

Die Chancen der Partnerin, am neuen Arbeitsort des Mannes eine Stelle zu finden, sind mittelmässig und die Verdienstmöglichkeiten der Partnerin sind im Vergleich zum hiesigen Arbeitsmarkt dort höher. Im Haushalt lebt ihr gemeinsames 3-jähriges Kind. Wenn das Paar nicht umzieht, würde ein einfacher Arbeitsweg für den Mann 11/2 Stunden dauern.

Wie gerecht finden Sie es, wenn die Partnerin vom Mann verlangt, er solle pendeln?

$\begin{array}{lllllllllll}\text { sehr gerecht } \mathrm{O} & \mathrm{O} & \mathrm{O} & \mathrm{O} & \mathrm{O} & \mathrm{O} & \mathrm{O} & \mathrm{O} & \mathrm{O} & \mathrm{O} & \mathrm{O} \text { sehr ungerecht }\end{array}$

Auf einer 11-stufigen Likertskala wurde die Antwort der Probanden erfasst, wie ungerecht sie es finden, wenn ALTER von EGO erwartet, dass EGO pendeln soll (siehe Abbildung 2). Ein Urteil ganz links auf der Skala wurde mit 1 eingestuft (sehr gerecht). Ein Urteil von ,sehr ungerecht' am anderen Ende der Skala wurde mit 11 codiert. Diese Gerechtigkeitsurteile der Befragten, welches Geschlecht in den Vignetten die Pendelkosten tragen soll, wird in der empirischen Analyse als abhängige Variable verwendet. Damit werden die Gerechtigkeitsvorstellungen der Probanden geschlechtsspezifisch zum Ausdruck gebracht. 


\section{Empirische Ergebnisse}

Tabelle 2 informiert über erste Resultate der abhängigen Variable. Alle Ungerechtigkeitsurteile streuen auf der Skala von 1 bis 11. Der Mittelwert liegt im Durchschnitt für alle Befragten und aufgesplittert in die Geschlechtergruppen der Befragten leicht über dem Skalenmittel von 6 Punkten. Die insgesamt 2839 Urteile der Probanden tendieren also allgemein etwas mehr dazu, in den Vignettensituationen eine Forderung von ALTER an EGO, zu pendeln, als ungerecht zu bewerten. Für die Beurteilung der Forderung von ALTER an EGO zu pendeln macht es bei den beiden befragten Geschlechtern keinen Unterschied, ob es sich bei EGO um einen Mann oder eine Frau handelt. ${ }^{13}$

Tabelle 2: Analyse der abhängigen Variable

\begin{tabular}{|c|c|c|c|c|c|c|c|c|}
\hline $\begin{array}{l}\text { Ungerechtigkeitsurteile: } \\
\text { Erwartung von ALTER an } \\
\text { EGO, zu Pendeln }\end{array}$ & $\begin{array}{l}\text { Anzahl } \\
\text { Urteile }\end{array}$ & Min & $\max$ & sd & $\begin{array}{l}\text { Mittel- } \\
\text { werte }\end{array}$ & $\begin{array}{l}\text { Diffe- } \\
\text { renz }\end{array}$ & $\begin{array}{l}\text { T- } \\
\text { Wert }\end{array}$ & $\begin{array}{l}\text { Signifi- } \\
\text { kanz }\end{array}$ \\
\hline \multicolumn{9}{|l|}{ Alle Befragten $N=356$} \\
\hline $\begin{array}{l}\text { falls EGO in Vignette }= \\
\text { Mann }\end{array}$ & 1881 & 1 & 11 & 3.15 & 6.350 & \multirow[t]{2}{*}{0.099} & \multirow[t]{2}{*}{0.789} & \multirow[t]{2}{*}{ keine } \\
\hline falls EGO in Vignette $=$ Frau & 958 & 1 & 11 & 3.20 & 6.250 & & & \\
\hline \multicolumn{9}{|l|}{ Befragte Frauen N=199 } \\
\hline $\begin{array}{l}\text { falls EGO in Vignette }= \\
\text { Mann }\end{array}$ & 1051 & 1 & 11 & 3.13 & 6.255 & \multirow[t]{2}{*}{-0.000} & \multirow[t]{2}{*}{-0.001} & \multirow[t]{2}{*}{ keine } \\
\hline falls EGO in Vignette $=$ Frau & 535 & 1 & 11 & 3.21 & 6.256 & & & \\
\hline \multicolumn{9}{|l|}{ Befragte Männer N=157 } \\
\hline $\begin{array}{l}\text { falls EGO in Vignette }= \\
\text { Mann }\end{array}$ & 830 & 1 & 11 & 3.18 & 6.469 & \multirow[t]{2}{*}{0.226} & \multirow[t]{2}{*}{1.187} & \multirow[t]{2}{*}{ keine } \\
\hline falls EGO in Vignette $=$ Frau & 423 & 1 & 11 & 3.20 & 6.243 & & & \\
\hline
\end{tabular}

Für die multivariate Analyse werden in Tabelle 3 erste Modelle präsentiert. Die erhobenen Daten besitzen aufgrund der Vignettenstruktur das Problem, dass die Annahme der Unabhängigkeit der einzelnen Fälle - hier also der 8 Vignetten pro Person - verletzt wird. Da jeder Befragte mehrere Vignetten beantwortet, werden die Eigenschaften des Befragten simultan mehrere Vignettenurteile beeinflussen. Um die dadurch verursachte Verzerrung der Schätzung zu korrigieren, greifen wir auf ein Random-Intercept-Modell zurück, das die Mehrebenenstruktur der Daten berücksichtigt (vgl. Auspurg / Abraham / Hinz 2009). Die hauptsächlich interessierende Variable in Tabelle 3 ist das Geschlecht von EGO in den Vignetten. Machen die Befragten einen Unterschied in ihrem Ungerechtigkeitsurteil, wenn es sich bei EGO um einen Mann oder eine Frau handelt? Der Koeffizient der Variable des Geschlechts von EGO hat in Modell 1 unter Kontrolle der restlichen mobilitätsrelevanten - und persönlichen Dimensionen den nicht signifikanten Wert von -0.057. Wie schon in Tabelle 2 erwähnt, machen die Befragten keine Unterschiede in ihrem Ungerechtigkeitsurteil, wenn es sich bei EGO um einen Mann oder eine Frau handelt. Auch der Koeffizient der Variable des Geschlechts der Befragten lässt in Modell 1 keinen signifikanten Geschlechtereffekt im Antwortverhalten erkennen (-0.114).

13 Die gleichmäßige Verteilung der abhängigen Variable kann zudem als Beweis dafür erachtet werden, dass die fraktionelle Auswahl der Vignetten und deren Verteilung auf die Decks gelungen ist. 
Tabelle 3: Modelle zur Überprüfung des Mobilitätsdilemmas und Hypothese 1

\begin{tabular}{|c|c|c|c|c|c|c|c|c|}
\hline $\begin{array}{l}\text { AV: Ungerechtigkeitsur- } \\
\text { teile (Skala 1-11) } \\
\text { Erwartung von ALTER } \\
\text { an EGO, zu Pendeln } \\
\text { (negativ=gerecht, posi- } \\
\text { tiv=ungerecht) }\end{array}$ & \multicolumn{2}{|c|}{$\begin{array}{l}\text { Grund- } \\
\text { modell } \\
\text { Modell } 1\end{array}$} & \multicolumn{2}{|c|}{$\begin{array}{l}\text { Interaktions- } \\
\text { modell } \\
\text { Modell } 2\end{array}$} & \multicolumn{2}{|c|}{$\begin{array}{l}\text { Befragte } \\
\text { Männer } \\
\text { Modell 2a }\end{array}$} & \multicolumn{2}{|c|}{$\begin{array}{l}\text { Befragte } \\
\text { Frauen } \\
\text { Modell } 2 \text { b }\end{array}$} \\
\hline \multicolumn{9}{|l|}{ Vignettenmerkmale } \\
\hline $\begin{array}{l}\text { EGO }(1=\text { falls EGO eine } \\
\text { Frau ist })\end{array}$ & -0.057 & & -0.299 & $*$ & -0.293 & $*$ & 0.135 & \\
\hline $\begin{array}{l}\text { Interaktion Vignetten- } \\
\text { geschlecht*Befragtenge- } \\
\text { schlecht }\end{array}$ & & & 0.435 & $*$ & & & & \\
\hline \multicolumn{9}{|l|}{ Kinder (Ref.: keine) } \\
\hline - Ja, 3-jährig & -0.048 & & -0.047 & & 0.010 & & -0.100 & \\
\hline - Ja, 8-jährig & -0.677 & $* * *$ & -0.680 & $* * *$ & -0.765 & $* * *$ & -0.624 & *** \\
\hline \multicolumn{9}{|l|}{ Pendelzeit (Ref.: $3 / 4$ Std.) } \\
\hline$-1 \frac{1}{2} \operatorname{Std}$ & 2.054 & $* * *$ & -0.680 & $* * *$ & 1.879 & $* * *$ & 2.198 & *** \\
\hline-3 Std. & 4.200 & $* * *$ & 2.056 & $* * *$ & 4.073 & $* * *$ & 4.301 & *** \\
\hline \multicolumn{9}{|l|}{$\begin{array}{l}\text { Beschäftigungsaussich- } \\
\text { ten Alter am Zielort } \\
\text { (Ref.: gut) }\end{array}$} \\
\hline - mittelmäßig & -0.967 & $* * *$ & -0.969 & $* * *$ & -0.870 & $* * *$ & -1.052 & $* * *$ \\
\hline - gering & -1.586 & $* * *$ & 1.586 & $* * *$ & -1.406 & $* * *$ & -1.728 & *** \\
\hline \multicolumn{9}{|l|}{$\begin{array}{l}\text { Verdienstaussichten Al- } \\
\text { ter am Zielort (Ref.: hö- } \\
\text { her) }\end{array}$} \\
\hline - vergleichbar & -0.538 & $* * *$ & -1.402 & $* * *$ & -0.509 & $* * *$ & -0.557 & *** \\
\hline - niedriger & -1.399 & $* * *$ & -0.970 & $* * *$ & -1.223 & $* *$ & -1.544 & $* * *$ \\
\hline \multicolumn{9}{|l|}{ Befragtenmerkmale } \\
\hline Geschlecht $(1=$ Frau $)$ & -0.114 & & -0.261 & + & & & & \\
\hline Geburtsort $(1=\mathrm{CH})$ & 0.061 & & 0.061 & & 0.193 & & -0.064 & \\
\hline $\begin{array}{l}\text { Höchster Bildungsab- } \\
\text { schluss Vater }\end{array}$ & 0.066 & & 0.065 & & 0.090 & & 0.065 & \\
\hline $\begin{array}{l}\text { Höchster Bildungsab- } \\
\text { schluss Mutter }\end{array}$ & -0.035 & & -0.035 & & -0.012 & & -0.063 & \\
\hline $\begin{array}{l}\text { Befrage in Beziehung } \\
(1=\text { ja) }\end{array}$ & -0.006 & & -0.006 & & -0.225 & & 0.149 & \\
\hline Konstante & 5.904 & $* * *$ & 5.989 & $* * *$ & 5.776 & $* * *$ & 5.895 & $* * *$ \\
\hline $\begin{array}{l}\text { Anzahl Fälle, Anzahl Ur- } \\
\text { teile }\end{array}$ & 348 & 2775 & 348 & 2775 & 154 & 1229 & 194 & 1546 \\
\hline Befragtenebene $\left(\sigma_{v}^{2}\right)$ & 1.097 & & 1.097 & & 1.093 & & 1.092 & \\
\hline Vignettenebene $\left(\sigma_{\varepsilon}^{2}\right)$ & 2.201 & & 2.198 & & 2.293 & & 2.112 & \\
\hline
\end{tabular}

Random-Intercept-Modelle (Maximum-Likelihood-Schätzung). Signifikant für: $p<0.001(* * *)$, $\mathrm{p}<0.01(* *), \mathrm{p}<0.05\left(^{*}\right), \mathrm{p}<0.1(+)$ bei zweiseitigem Test. 
In einem ersten Schritt soll überprüft werden, in welchem Ausmaß die Befragten durch ihre Gerechtigkeitsurteile tatsächlich eine Konfliktsituation zwischen ALTER und EGO in den Vignetten zum Ausdruck bringen oder ob dieser Konflikt bereits durch geschlechtsspezifische Normen abgemildert wird. Zur Überprüfung dieser Aussage wurde in Modell 2 das Geschlecht von EGO mit dem Geschlecht der Befragten interagiert. Gefragt wird danach, ob bei der Erwartung, welches Geschlecht in den Vignetten die Pendelkosten tragen soll, Geschlechtereffekte im Antwortverhalten der Befragten auftreten.

Der Interaktionsterm $\left(0.435^{*}\right)$ bestätigt einen diesbezüglich signifikanten Unterschied. Der Haupteffekt des Geschlechts von EGO (-0.299*) gilt im Interaktionsmodell 2 nur für die Gruppe, welche in der Moderatorvariable des Befragtengeschlechts den Wert Null hat - in diesem Fall die befragten Männer. Dieses Urteil der männlichen Studenten fällt signifikant weniger ungerecht aus, wenn es sich bei EGO um eine Frau und nicht um einen Mann handelt. Der Koeffizient des Geschlechts der Befragten von -0.261+ zeigt seinerseits einen auf dem $10 \%$ Niveau signifikanten Effekt. Er besagt, dass sich die befragten Frauen im Vergleich zu den befragten Männern in ihrem Urteil darüber, ob in den Vignetten EGO-Männer die Pendelkosten tragen sollen, mit einer Wahrscheinlichkeit von $90 \%$ unterscheiden. Etwas übersichtlicher sind dieselben Effekte in Tabelle 3.1 dargestellt.

Tabelle 3.1: Interaktionseffekt Geschlecht von EGO*Geschlecht der Befragten (Modell 2)

\begin{tabular}{|l|l|l|l|}
\hline \multicolumn{2}{|l|}{ Ungerechtigkeitsurteile } & \multicolumn{2}{c|}{ Geschlecht von EGO } \\
\cline { 3 - 4 } & \multicolumn{1}{|c|}{ Mann $=0$} & Frau $=1$ \\
\hline $\begin{array}{l}\text { Befragten- } \\
\text { geschlecht }\end{array}$ & Mann $=0$ & Referenzkategorie & $\beta_{2}=-0.299 *$ \\
\cline { 2 - 4 } & Frau $=1$ & $\beta_{1}=-0.261+$ & $\beta_{3}=0.435 *$ \\
\hline
\end{tabular}

Das Fazit dieser Analyse des Mobilitätsdilemmas ist, dass bei einem arbeitsmarktbedingten Mobilitätsanreiz eines Partners Pendeln von den Probanden dann tendenziell eher als ungerecht empfunden wird, wenn das Arbeitsangebot in den Vignetten das jeweils eigene Geschlecht betrifft. Befragte Männer und Frauen solidarisieren sich also in ihrem Antwortverhalten mit den je eigenen Geschlechterkategorien in den Vignetten.

„, To the extent that norms differ for women and men, people tend to refer to what is desirable for individuals of their own sex and thereby form intensions and engage in actions that differ by sex" (Eagly / Wood / Diekman 2000: 131). Dieses Phänomen der Solidarisierung mit den je eigenen Geschlechterkategorien, stützen auch weitere Ergebnisse in der Sozialpsychologie (vgl. Grossman / Wood 1993).

Abbildung 3 verdeutlicht noch einmal den signifikanten Unterschied zwischen den befragten Männern und Frauen bezüglich der Beurteilung, welches Geschlecht in den Vignetten die Pendelkosten tragen soll. ${ }^{14}$ Die Gerechtigkeitsurteile der Probanden besagen, dass bei zwei

14 Der Plot wurde so generiert, dass aus den Regressionskoeffizienten pro Modell getrennt nach EGO Geschlecht die Wahrscheinlichkeit berechnet wurde, ob eher EGO-Frauen oder EGO-Männer die Pendelkosten übernehmen sollen. Die übrigen Variablen in den Modellen enthalten jeweils Mittelwertcharakteristiken. 
Partnern mit vergleichbaren Humankapitalressourcen in einem Haushalt bei einem arbeitsmarktbedingten Mobilitätsanreiz eines Partners, das jeweils andere Geschlecht pendeln soll.

Abbildung 3: Wahrscheinlichkeitsplots, getrennt nach dem Geschlecht der Befragten (Modelle 2)

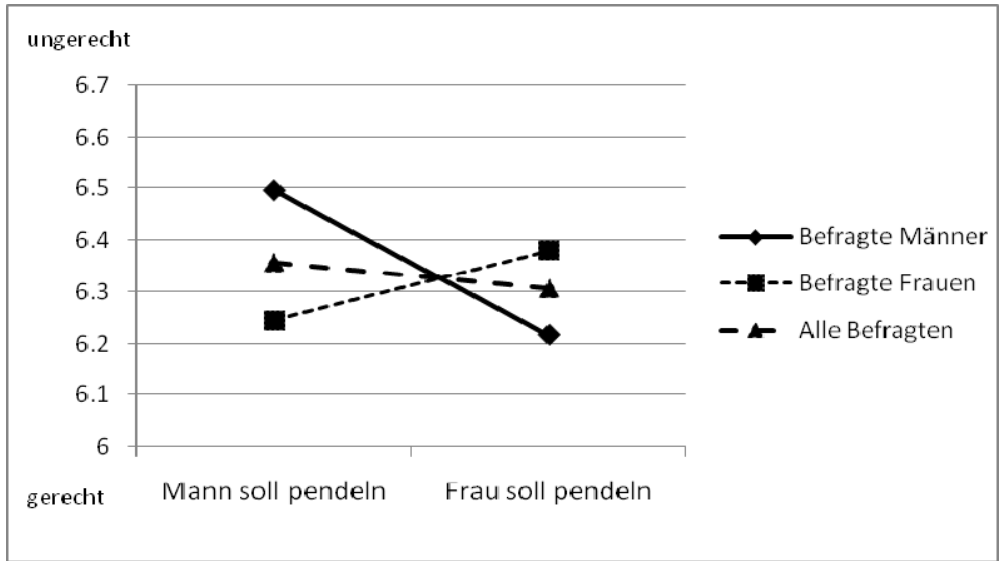

Mit den Modellen 2 lässt sich auch gleich beantworten, ob die Befragten in ihrem Antwortverhalten von traditionellen Rollenvorstellungen ausgehen. Wenn Hypothese 1 zutrifft, würden die befragten Männer und Frauen angeben, dass es in den Vignetten jeweils die Frauen sind, die in ihrer Mobilitätspräferenz nachgeben sollten. Zur besseren Übersicht sind in den Modellen $2 \mathrm{a}$ und $2 \mathrm{~b}$ die Resultate der Geschlechtergruppen getrennt dargestellt. Modell $2 \mathrm{a}$ zeigt noch einmal die im Interaktionsmodell beschriebene Aussage der befragten Männer, dass sie es ungerecht finden, wenn Männer in den Vignetten in ihrer Mobilitätspräferenz nachgeben müssten $\left(-0.293^{*}\right)$. Der Koeffizient des Geschlechts von EGO für die befragten Frauen von Modell $2 \mathrm{~b}$ hingegen ist stark positiv, wenn auch nicht signifikant. Tendenziell beurteilen es Probandinnen als ungerecht, wenn Frauen in den Vignetten die Pendelkosten übernehmen sollen. Wie das Interaktionsmodell 2 gezeigt hat, gehen die Aussagen der befragten Geschlechter mit einem Signifikanzniveau von 10\% in entgegen gesetzte Richtungen $(-0.261+)$. Die Annahme der Existenz einer traditionellen Geschlechternorm für zwei Partner mit vergleichbaren haushalts- und arbeitsmarktrelevanten Kriterien kann somit nicht empirisch gestützt werden.

Bei den übrigen mobilitätsrelevanten Variablen in Modell 1 und 2 hat, wenig überraschend, die Länge des Arbeitsweges den stärksten Einfluss. Je länger der Weg dauert, desto ungerechter wird von allen Befragten die Forderung von ALTER eingeschätzt, dass EGO pendeln soll. Weitere starke Einflüsse in den Vignetten sind die Dimensionen der Arbeitsmarktchancen von ALTER am neuen Arbeitsort von EGO. Je schlechter die Beschäftigungs- und Verdienstaussichten von ALTER am neuen Ort von EGO sind, umso weniger ungerecht beurteilen die Befragten die Erwartung von ALTER, dass EGO pendeln soll. Auch der Koeffizient für schulpflichtige Kinder fällt im Vergleich mit Vignettenpaaren ohne Kinder in den Vignetten signifikant negativ aus. Sind die Kinder hingegen noch im Vorschulalter, ist das Ungerechtigkeitsurteil nicht signifikant unterschiedlich im Vergleich mit Paaren, die keine Kinder haben. Dieser Befund lässt sich dahingehend deuten, dass es für Paare mit schulpflichtigen Kindern schwieriger ist, den Wohnsitz zu verlegen (weshalb Pendeln für EGO auch als weniger ungerecht beurteilt wird, als für Paare ohne oder für Paare mit Kindern im Vorschulalter). Demzufolge 
wird bei den Befragten Pendeln von EGO eher als wünschenswert eingestuft. Bei den Befragtenmerkmalen gibt es außer dem Geschlecht der Probanden kein Merkmal, das zur Erklärung dafür herangezogen werden könnte, welcher der Partner in den Vignetten die Pendelkosten tragen sollte. ${ }^{15}$

Im Folgenden soll nun überprüft werden, ob eine Norm über die Zuständigkeit der Kinderbetreuung die symmetrischen Machtverhältnisse von Paaren verschieben kann, damit sie eine Lösung aus dem Mobilitätsdilemma finden. Wenn Hypothese 2 zutrifft, wären gemäß der sozialen Norm vor allem Frauen für die Kinderbetreuung zuständig und folglich die Männer diejenigen, die bei einem arbeitsmarktbedingten Mobilitätsanreiz in ihrer Mobilitätspräferenz nachgeben würden. Mit Tabelle 4 werden die Ergebnisse zu Hypothese 2 diskutiert, wobei zur besseren Vergleichbarkeit Modell 1 in Tabelle 4 identisch ist mit Modell 1 in Tabelle 3. In Tabelle 4 wird zudem, mit Ausnahme der Variable des Befragtengeschlechts, auf die Darstellung der restlichen Befragtenmerkmale verzichtet. Wie in Tabelle 3, sind diese allesamt nicht signifikant. In Modell 3 werden das Geschlecht von EGO und die Variable der Kinder als Interaktion geschätzt. Sind 3-jährige Kinder im Haushalt, wird es von den Befragten als signifikant ungerecht beurteilt $\left(0.853^{* * *}\right)$, wenn weibliche EGOs im Vergleich zur Referenzkategorie ,Frauen in Haushalten ohne Kinder' pendeln müssten. Sind die Kinder 8-jährig, ist das Ergebnis nicht mehr signifikant. Der Koeffizient von 0.364 zeigt für alle Befragten tendenziell zwar noch in dieselbe Richtung. Wenn die Kinder jedoch im Schulalter sind, scheint es weder gerecht noch ungerecht zu sein, dass Frauen die Pendelkosten tragen. Diese Interaktionsergebnisse bestätigen sich auch, wenn beide befragten Geschlechter getrennt geschätzt werden. Sowohl die Gruppe der befragten Männer (Modell 3 a) als auch die Gruppe der befragten Frauen (Modell 3 b) beurteilen es als ungerecht, wenn Frauen mit 3-jährigen Kindern zusätzlich zur Kinderbetreuung noch die Pendelkosten tragen sollen. Für Paare in den Vignetten mit 8-jährigen Kindern hingegen unterscheiden sich die Urteile der Probanden nicht mehr signifikant von Paaren ohne Kinder.

Der Koeffizient des Geschlechts von EGO in Modell $3\left(-0.471^{* *}\right)$ gilt wiederum nur falls die Moderatorvariable Null ist - in diesem Fall Paare ohne Kinder. Sind keine Kinder im Haushalt, wird es von allen Befragten als signifikant weniger ungerecht beurteilt, wenn Frauen pendeln sollen. Ein Blick in die geschlechtergetrennten Modelle zeigt, dass dieses Ergebnis vor allem für die Gruppe der befragten Männer gilt. Die befragten Frauen sind in ihrer Aussage darüber, wer in einem kinderlosen Haushalt die Pendelkosten übernehmen soll, indifferent. Die restlichen mobilitätsrelevanten Vignettenmerkmale tragen in etwa gleich viel zur Erklärung der abhängigen Variable bei wie schon in Tabelle 3. Hypothese 2 bestätigt sich somit nur für EGO-Frauen, die Mütter von vorschulpflichtigen Kindern sind. Diese Frauen sollen, nach den Urteilen der befragten Studierenden, nebst der Kinderbetreuung nicht auch noch die Pendelkosten tragen müssen. Gesellschaftliche Normvorstellungen darüber, wer für die Betreuung von vorschulpflichtigen Kindern zuständig ist, könnten somit das Machtverhältnis zwischen zwei anfänglich ebenbürtigen Partnern verschieben. Kommen Frauen ihrer gesellschaftlich zugedachten Mutterrolle nach, wird es als gerecht beurteilt, wenn die Pendelkosten zulasten der Väter gehen.

15 Erwähnenswert ist noch, dass - bis auf den Geschlechtereffekt - alle mobilitätsrelevanten Faktoren in den Vignetten von beiden befragten Geschlechtergruppen mit vergleichbaren Kosten- und Nutzenabwägungen bewertet wurden (vgl. Modelle $2 \mathrm{a}$ und $2 \mathrm{~b}$ ). Dies kann zusätzlich als Indiz aufgefasst werden, dass sich die Befragten für ihr Antwortverhalten an ihrem je eigenen Geschlecht in den Vignetten orientiert haben. 
Tabelle 4: Modelle zur Überprüfung von Hypothese 2

\begin{tabular}{|c|c|c|c|c|c|c|c|c|}
\hline $\begin{array}{l}\text { AV: Ungerechtig- } \\
\text { keitsurteil (Skala } \\
\text { 1-11) } \\
\text { Erwartung von AL- } \\
\text { TER an EGO zu Pen- } \\
\text { deln } \\
\text { (negativ=gerecht, po- } \\
\text { sitiv=ungerecht) }\end{array}$ & \multicolumn{2}{|c|}{$\begin{array}{l}\text { Grundmodell } \\
\text { Modell } 1\end{array}$} & \multicolumn{2}{|c|}{$\begin{array}{c}\text { Interaktions- } \\
\text { modell } \\
\text { Modell } 3\end{array}$} & \multicolumn{2}{|c|}{$\begin{array}{l}\text { Interaktion } \\
\text { befr. Männer } \\
\text { Modell } 3 \text { a }\end{array}$} & \multicolumn{2}{|c|}{$\begin{array}{l}\text { Interaktion } \\
\text { befr. Frauen } \\
\text { Modell 3 b }\end{array}$} \\
\hline \multicolumn{9}{|l|}{ Vignettenmerkmale } \\
\hline $\begin{array}{l}\text { EGO-Geschlecht }(1= \\
\text { falls EGO eine Frau } \\
\text { ist) }\end{array}$ & -0.057 & & -0.471 & $* *$ & -0.752 & $* * *$ & -0.239 & \\
\hline \multicolumn{9}{|l|}{ Kinder (Ref.: keine) } \\
\hline - Ja, 3-jährig & -0.048 & & -0.333 & $*$ & -0.282 & & -0.384 & $*$ \\
\hline -Ja, 8-jährig & -0.677 & $* * *$ & -0.801 & $* * *$ & -0.929 & $* * *$ & -0.708 & $* * *$ \\
\hline \multicolumn{9}{|l|}{$\begin{array}{l}\text { Interaktion } \\
\text { EGO-Ge- } \\
\text { schlecht*Kinder } \\
\text { (Ref.: keine) }\end{array}$} \\
\hline -Ja, 3-jährig & & & 0.853 & $* * *$ & 0.860 & $*$ & 0.862 & $* *$ \\
\hline -Ja, 8-jährig & & & 0.364 & & 0.481 & & 0.247 & \\
\hline \multicolumn{9}{|l|}{$\begin{array}{l}\text { Pendelzeit (Ref.: } 3 / 4 \\
\text { Std.) }\end{array}$} \\
\hline$-1 \frac{1}{2}$ Std. & 2.054 & $* * *$ & 2.066 & $* * *$ & 2.201 & $* * *$ & 2.218 & $* * *$ \\
\hline-3 Std. & 4.200 & $* * *$ & 4.206 & $* * *$ & 4.078 & $* * *$ & 4.310 & $* * *$ \\
\hline \multicolumn{9}{|l|}{$\begin{array}{l}\text { Beschäftigungsaus- } \\
\text { sichten Alter am Ziel- } \\
\text { ort (Ref.: gut) }\end{array}$} \\
\hline - mittelmäßig & -0.967 & $* * *$ & -0.985 & $* * *$ & -0.885 & $* * *$ & -1.072 & $* * *$ \\
\hline - gering & -1.586 & $* * *$ & -1.604 & $* * *$ & -1.421 & $* * *$ & -1.749 & $* * *$ \\
\hline \multicolumn{9}{|l|}{$\begin{array}{l}\text { Verdienstaussichten } \\
\text { Alter am Zielort (Ref.: } \\
\text { höher) }\end{array}$} \\
\hline - vergleichbar & -0.538 & $* * *$ & -0.528 & $* * *$ & -0.489 & $* *$ & -0.556 & $* * *$ \\
\hline - niedriger & -1.399 & $* * *$ & -1.399 & $* * *$ & -1.218 & $* * *$ & -1.549 & $* * *$ \\
\hline $\begin{array}{l}\text { Befragten Geschlecht } \\
(1=\text { Frau })\end{array}$ & -0.114 & & -0.112 & & & & & \\
\hline $\begin{array}{l}\text { Anzahl Fälle, Anzahl } \\
\text { Urteile }\end{array}$ & 348 & 2775 & 348 & 2775 & 154 & 1229 & 194 & 1546 \\
\hline Befragtenebene $\left(\sigma_{v}^{2}\right)$ & 1.097 & & 1.089 & & 1.093 & & 1.077 & \\
\hline Vignettenebene $\left(\sigma_{\varepsilon}^{2}\right)$ & 2.201 & & 2.196 & & 2.288 & & 2.108 & \\
\hline
\end{tabular}

Random-Intercept-Modelle (Maximum-Likelihood-Schätzung). Signifikant für: $p<0.001(* * *)$, $\mathrm{p}<0.01\left(^{* *}\right), \mathrm{p}<0.05\left(^{*}\right), \mathrm{p}<0.1(+)$ bei zweiseitigem Test. 


\section{Diskussion}

Die dual earner Familie ist mittlerweile in modernen Gesellschaften die vorherrschende Haushaltsform (vgl. Gershuny / Bittman / Brice 2005). Partner von Zweiverdienerhaushalten sind herausgefordert zwei Arbeitsorte und einen Wohnort zu koordinieren. Vor allem ein Stellenwechsel eines Partners an einen entfernten Ort kann die gemeinsame Beziehung stark belasten. Können sich die Partner nicht über einen gemeinsamen Umzug einigen, stellt sich die Frage; wer soll pendeln?

Ausgehend von einem abstrakten Modell wurde eine Situation konstruiert, in der die Partner gegensätzliche Interessen bezüglich einer Mobilitätsentscheidung besitzen. Im Rahmen dieses Modells präferiert jeder Partner, dass der jeweils andere die Pendelkosten trägt. Aufgrund der gegenläufigen Präferenzen der Partner sollte sich bei annähernd symmetrischer Verhandlungsmacht ein grundsätzlicher Konflikt ergeben, dessen Lösung ein Nachgeben einer Seite erfordert. Stellen allgemeine gesellschaftliche Normen einen Lösungsmechanismus für diese Situation dar? Um diese Frage zu klären, wurden Probanden gebeten die Gerechtigkeit einer vorgegebenen Lösung einzuschätzen. Einerseits konnte so überprüft werden, ob die modellierten Situationen tatsächlich als Konflikt wahrgenommen wurden und andererseits welche Faktoren welche Lösung des Dilemmas begünstigen.

Ein grundsätzlicher Mobilitätskonflikt zwischen zwei erwerbstätigen Partnern mit gemeinsamem Wohnsitz konnte nachgewiesen werden. Weiter wurde gezeigt, dass dieser Konflikt nicht durch eine allgemeingültige Geschlechternorm gelöst werden kann. Zwei annähernd ebenbürtige Partner orientieren sich für ihre Mobilitätsentscheidungen nicht an gesellschaftlich vorgegebenen Rollenbildern eines männlichen ,mainbreadwinners' und einer weiblichen ,housekeeperin'. Hingegen kann die Existenz von Kindern die Partner aus dem Mobilitätsdilemma führen. Es scheint eine gesellschaftliche Norm zu bestehen, dass Frauen primär für die Kleinkinderbetreuung zuständig sind und es deshalb ungerecht wäre, wenn sie auch noch die Pendelkosten zu tragen hätten. Die Kosten der Väter als ,tied commuter' sind folglich geringer einzuschätzen als die zu erwartenden Pendelkosten für die Mütter. Sind die Kinder jedoch im Schulalter bleibt der Mobilitätskonflikt bestehen. Diese Befunde stützen die allgemein bekannten Ergebnisse aus der Familiensoziologie, dass (Klein)kinder der zentrale Auslöser für eine Umorganisation der Partnerschaft darstellen (Gornick / Meyers 2005; Peukert 2005; Burkart 2008).

Offen bleibt somit die Frage, wie Paare mit zwei erwerbstätigen Partnern einen Mobilitätskonflikt umgehen können, wenn sie Kinder im Schulalter oder gar keine Kinder haben. Die normativen Urteile der Probanden zu gerechten Mobilitätsarrangements trugen diesbezüglich wenig zur Klärung bei. Zu vermuten ist, dass Paare mit Kindern im Schulalter oder gar keinen Kindern den Mobilitätskonflikt gar nicht zu lösen versuchen, weil die Ablehnung durch einen Partner schon zum vornherein antizipiert würde (vgl. Kalter 1998; Schneider / Limmer / Ruckdeschel 2002). Wie die Arbeitsmarktforschung zeigt, ist ein Nichteintreten auf den Mobilitätskonflikt für die Arbeitsmarktplatzierung der Partner aber unvorteilhaft, weil der, MatchingProzess` bei hoher regionaler Mobilität von Arbeitskräften wesentlich leichter gelöst werden kann als bei Immobilität (Franz 2006). Welche Strategien Doppelverdiener mit Schulkindern oder ohne Kinder helfen, eine Lösung aus dem Mobilitätsdilemma zu finden bleibt Gegenstand weiterer Untersuchungen. 


\section{Literatur}

Abraham, Martin / Katrin Auspurg / Thomas Hinz (2010): Migration Decisions Within Dual-Earner Partnerships: A Test of Bargaining Theory, in: Journal of Marriage and the Family 72, S. 876-892.

Abraham, Martin/ Thomas Hinz (2005): Arbeitsmarktsoziologie. Probleme, Theorien, empirische Befunde, Wiesbaden.

Abraham, Martin / Thess Schönholzer (2009): Pendeln oder Umziehen? Entscheidungen über unterschiedliche Mobilitätsformen in Paarhaushalten, in: Gross, Christiane / Peter Kriwy (Hrsg.): Klein aber fein! Quantitative empirische Sozialforschung mit kleinen Fallzahlen. Wiesbaden, S. 227-248.

Auspurg, Katrin / Martin Abraham / Thomas Hinz (2009): Die Methodik des Faktoriellen Surveys in einer Paarbefragung, in: Gross, Christiane / Peter Kriwy (Hrsg.): Klein aber fein! Quantitative empirische Sozialforschung mit kleinen Fallzahlen. Wiesbaden, S. 179-210.

Auspurg, Katrin / Thomas Hinz / Stefan Liebig / Carsten Sauer (2008): Wer verdient welches Einkommen? Ergebnisse eines faktoriellen Surveys zur Einkommensgerechtigkeit in Deutschland. Working Paper \# 3 des DFG-Projekts „Der faktorielle Survey als Instrument zur Einstellungsmessung in Umfragen“. Universität Bielefeld, Universität Konstanz.

Baldridge, David C. / Kimberly A.Eddleston / John F. Veiga (2006): Saying 'no' to being uprooted: The impact of family and gender on willingness to relocate, in: Journal of Occupational and Organizational Psychology 79, S. 131-149.

Beck, Michael / Karl-Dieter Opp (2001): Der faktorielle Survey und die Messung von Normen, in: Kölner Zeitschrift für Soziologie und Sozialpsychologie 53, S. 283-306.

Bielby William T. / Denise D. Bielby (1992): I Will Follow Him: Family Ties, Gender-Role Beliefs, and Reluctance to Relocate for a Better Job, in: American Journal of Sociology 97, S. 1241-1267.

Bundesamt für Statistik (2005): Haushalte und Familien. Neuchâtel.

Bühler, Elisabeth / Corinna Heye (2005): Fortschritte und Stagnation in der Gleichstellung der Geschlechter 1970-2000. Neuchâtel.

Burkart, Günter (2008): Familiensoziologie. Konstanz.

Carnazzi Weber, Sara / Sylvie Golay (2005): Interne Migration in der Schweiz. Neuchâtel.

Clark, William A. V. / Youqin Huang / Suzanne Withers (2003): Does commuting distance matter? Commuting tolerance and residential change, in: Regional Science and Urban Economics 33, S. 199-221.

Dülmer, Hermann (2007): Experimental Plans in Factorial Surveys. Random or Quota Design? in: Sociological Methods \& Research, 35, S. 382-409.

Eagly, Allice H. / Wendy Wood / Amanda B. Diekman (2000): Social role theory of sex differences and similarities: A current appraisal, in: Eckes T. / H. M. Trautner (Hrsg.), The Developmental Social Psychology of Gender. Mahwah, NJ, S. 123-174.

Elster, Jon (1989): Nuts and Bolts for the Social Sciences, Cambridge.

Franz, Wolfgang (2006): Arbeitsmarktökonomik. 6. Auflage, Berlin.

Frick, Roman / Philipp Wüthrich / René Zbinden/ Mario Keller (2004): Pendlermobilität in der Schweiz - Eidgenössische Volkszählung, Neuchâtel.

Gershuny, Jonathan / Michael Bittman / John Brice (2005): Exit, Voice and Suffering: Do Couples Adapt to Changing Employment Patterns? Journal of Marriage and Family 67, S. 656-665.

Gornick, Jante C. / Marcia K. Meyers (2005): Families That Work. Policies for Reconciling Parenthodd and Employment, New York.

Grossman, Michele / Wendy Wood (1993): Sex Differences in Intensity of Emotional Experience: A Social Role Interpretation, in: American Psychological Association 65, S. 1010-1022.

Holler, Manfred Joseph (2009): Einführung in die Spieltheorie, Berlin. 
Johnston-Anumonwo, Ibipo (1992): The influence of household type on gender differences in work trip distance, in: The Professional Geographer 44, S. 161-169.

Jürgens, Hendrik (1998): Beruflich bedingte Umzüge von Doppelverdienern, in: Zeitschrift für Soziologie 27, S. 358-377.

Jürgens, Hendrik (2005): The Geographic Mobility of Dual-Earner Couples: Do Gender Roles Matter? in: DIW Discussion Papers 474, Berlin.

Kalter, Frank (1994): Pendeln statt Migration. Die Wahl und Stabilität von Wohnort-Arbeitsort-Kombinationen, in: Kölner Zeitschrift für Soziologie und Sozialpsychologie 3, S. 460-476.

Kalter, Frank (1997): Wohnortswechsel in Deutschland. Ein Beitrag zur Migrationstheorie und zur empirischen Anwendung von Rational-Choice-Modellen, Opladen.

Kalter, Frank (1998): Partnerschaft und Migration. Zur theoretischen Erklärung eines empirischen Effekts, in: Kölner Zeitschrift für Soziologie und Sozialpsychologie 50, S. 283-309.

Liebig, Stefan / Steffen Mau (2002): Einstellungen zur sozialen Mindestsicherung. Ein Vorschlag zur differenzierten Erfassung normativer Urteile, in: Kölner Zeitschrift für Soziologie und Sozialpsychologie 54, S. 109-134.

Liebig, Stefan (2007): Modelle und Befunde der emprischen Gerechtigkeitsforschung in Deutschland am Beispiel der Einkommens- und Steuergerechtigkeit. In Stefan Empter und Robert B. Verkamp (Hrsg.): Soziale Gerechtigkeit - eine Bestandesaufnahme. Bertelsmann Stiftung, Gütersloh, S. 111-135.

Mincer, Jacob (1978): Family Migration Decisions, in: Journal of Political Economy 86, S. 749-773.

Nisic, Natascha (2009): Labour Market Outcomes of Spatially Mobile Coupled Women: Why is the Locational Context Important? In: Schmollers Jahrbuch (Journal of Applied Social Science Studies) 129, S. 1-13.

Nivalainen, Satu (2004): Determinants of family migration: short moves vs. long moves, in: Journal of Population Economics 17, S. 157-175.

Ommeren, Jos N. van / Piet Rietveld / Peter Nkjkamp (1997): Commuting: In Search of Jobs and Residences, in: Journal of Urban Economics 42, S. 402-421.

Ommeren, Jos N. van / Piet Rietveld / Peter Nkjkamp (1998): Spatial moving behavior of two-earner households, in: Journal of regional science 38, S. 23-41.

Ommeren, Jos N. van / Piet Rietveld / Peter Nkjkamp (1999): Job Moving, residential Moving, and Commuting: A Search Perspective, in: Journal of Urban Economics 46, S. 230-253.

Ott, Notburga (1989): Familienbildung und familiale Entscheidungsfindung aus verhandlungstheoretischer Sicht, in: Wagner, Gert / Notburga Ott / Hans-Joachim Hoffmann-Nowotny (Hrsg.), Familienbildung und Erwerbstätigkeit im demographischen Wandel. Berlin.

Ott, Notburga (1992): Intrafamily Bargaining and Houshold Decisions, Berlin u.a.

Ott, Notburga (1998): Der familienökonomische Ansatz von Gary S. Becker. S. 63-90: In: Ingo Pies und Martin Leschke (Hrsg). Gary Beckers ökonomischer Imperialismus. Tübingen: Mohr Siebeck.

Peukert, Rüdiger (2005): Familienformen im sozialen Wandel, 6. Aufl., Wiesbaden.

Rieck, Christian (2006): Spieltheorie. Eine Einführung, 6. Auflage, Eschborn.

Schelling, Tom (1960): The strategy of conflict. Cambridge.

Schneider, Norbert F. / Ruth Limmer / Kerstin Ruckdeschel (2002): Mobil, flexibel, gebunden. Familie und Beruf in der mobilen Gesellschaft, Frankfurt.

Statistisches Bundesamt (2002). Statistisches Jahrbuch 2002. Wiesbaden.

Turner, Tracy / Debbie Niemeier (1997): Travel to work and household responsibility: new evidence, in: Transportation 24, S. 397-419. 
Voss, Thomas (2001): Game-Theoretical Perspectives on the Emergence of Social Norms, in: Hechter, Michael / Karl-Dieter Opp (Hrsg.), Social Norms, New York.

Weinberger, Rachel (2005): Is shorter still better? An updated analysis of gender, race, and industrial segregation in San Francisco Bay Area commuting patterns, TRB 2006 Annual Meeting.

Thess Schönholzer Universität Bern

Institut für Soziologie Unitobler-Lerchenweg 36 3000 Bern 9 thess.schoenholzer@soz.unibe.ch 